\title{
Head and Neck Congenital Anomalies in Neonate Hospitals in Hail, Saudi Arabia
}

\author{
Ali A Assiry ${ }^{1}$, Siraj D Khan ${ }^{2}$, Hasna R Al-Shubrmi ${ }^{3}$, Dalal H Al-Shammary ${ }^{4}$, Hanan M Al-Fahhad ${ }^{5}$, Amnah F Al-Shammary ${ }^{6}$, \\ Tayf N Al-Shammari ${ }^{7}$, Abdullah F Al-Shammari ${ }^{8}$
}

\begin{abstract}
Introduction: There is secrecy in the literature published about congenital anomalies of head and neck. Dentists may encounter with many head and neck anomalies during their practice. This study aimed to assess the prevalence of head and neck anomalies among all congenital anomalies and to identify risk factors associated with head and neck anomalies.

Materials and methods: A cross-sectional hospital-based study was conducted on neonate data available in the records of neonate hospitals in Hail; records from 1432 to 1438 in Hijri Islamic calendar (equivalent to 2010-2016) were employed. All neonates from birth to 28 days after, who were born or admitted to these hospitals, were included in this study.

Results: The prevalence of head and neck anomalies among all congenital anomalies was 5.5\%. The highest incidence rate was found in year 1435 (Islamic Hijri Year), in which the incidence rate was $7.1 \%$. Cross tabulation between demographic characteristics and head and neck anomalies revealed that sex of the neonates was significantly associated with head and neck anomalies. The birth weight of the surveyed neonates and consanguinity marriage of the parents had no significant association with head and neck anomalies.

Conclusion: The occurrence of head and neck anomalies showed a wide variation in different years, and the prevalence among other anomalies is associated significantly with sex of newborn.

Keywords: Anomaly, Head and neck, Prevalence.

International Journal of Clinical Pediatric Dentistry (2020): 10.5005/jp-journals-10005-1734
\end{abstract}

\section{INTRODUCTION}

A congenital disorder also known as a genetic disease is a disease that occurs at or before birth irrespective of its origin. Among such conditions, structurally deformed conditions are referred as "congenital anomalies" which include defects in a developing fetus. ${ }^{1}$ Birth defects are extremely variable in cause and symptoms. Any substance, which causes birth defects, is referred to as a teratogen. Prenatal testing (screening) prior to birth can identify most of the disorders. Birth defects may result from factors related to genetics or the environment. It includes morphogenesis defects, infection, epigenetic modification to a parental germ line, or abnormalities in chromosome. The outcome of any congenital disorder depends not only on prenatal deficiencies but also on the postnatal environment. ${ }^{1}$ One of the major causes of stillbirth and neonatal mortality is congenital anomalies. The pattern and prevalence of congenital anomalies can differ over time or by location. ${ }^{2}$

Congenital head and neck malformations are wide and heterogeneous, as this region comprises part of all organ systems. Such malformations vary merely from cosmetic defects and mild disturbance to deadly abnormalities in their significance and severity. These can be isolated as a part of sequence, syndrome, or chromosomal disorder. Most of them are genetic in origin, but the rest are caused by teratogens. ${ }^{3}$

The prevalence of congenital anomalies in Saudi Arabia is widely known. Knowing the prevalence of congenital abnormalities and their pattern is potential in identifying causative or preventive factors. ${ }^{4}$ Some of the anomalies may be encountered by the practicing dentist, especially those of head and neck. ${ }^{5}$ The incidence of congenital abnormalities in children with low birth weight (LBW) and consanguineous marriages is much higher than average birth weight and non-consanguineous marriages. Although in

\footnotetext{
1,2Department of Preventive Dental Science, Division of Pediatric Dentistry, College of Dentistry, Najran University, Najran, Kingdom of Saudi Arabia

3,5,6 Ministry of Health, Hail, Kingdom of Saudi Arabia

${ }^{4}$ Endodontic Board Resident, Hail, Kingdom of Saudi Arabia

${ }^{7,8}$ College of Dentistry, University of Hail, Kingdom of Saudi Arabia
}

Corresponding Author: Siraj D Khan, Department of Preventive Dental Science, Division of Pediatric Dentistry, College of Dentistry, Najran University, Najran, Kingdom of Saudi Arabia, Phone: +966 532376159, e-mail:drsirajpedo@gmail.com

How to cite this article: Assiry AA, Khan SD, Al-Shubrmi HR, et al. Head and Neck Congenital Anomalies in Neonate Hospitals in Hail, Saudi Arabia. Int J Clin Pediatr Dent 2020;13(2):160-162.

Source of support: Nil

Conflict of interest: None

Saudi Arabia marriages are done at earlier ages, the women seek to give birth after the age of 36 years old, which increases the probability of having a baby with congenital anomalies. In addition, consanguineous marriage is common among Saudi families and aggravates the problem and was reported to increase the incidences of congenital anomalies. ${ }^{6,7}$

There is secrecy in the literature published about congenital anomalies of head and neck. Dentists may encounter with many head and neck anomalies during their practice. This study can provide baseline data on the identification of head and neck anomalies in Saudi Arabia. This study was conducted to assess the prevalence of head and neck anomalies among all congenital anomalies and to identify risk factors associated with head and neck anomalies. 


\section{Materials and Methods}

A cross-sectional hospital-based study was conducted on neonate data available in the records of neonate hospitals in Hail; records from 1432 to 1438 in Hijri Islamic calendar (equivalent to 2010-2016) were employed. All neonates from birth to 28 days after, who were born or admitted to these hospitals, were included in this study. Data from mothers as well as the different types of congenital anomalies of all body systems will be retrieved meticulously. Descriptive statistics for demographics of the studied newborns were analyzed using Statistical Package of Social Sciences version 20. The significant associations between congenital anomalies and those demographic characteristics were detected using Chisquare tests. The aims of this study were explained to the parents of newborns with assurance of confidentiality, and they provided written consents to participate in this survey. Ethical approval for this study was approved by the ethical committee in the College of Dentistry, Najran University.

\section{Results}

Out of 930 neonates who were included in this study, 896 neonates had complete data about their sex and $59.1 \%$ of them were males. The neonates were categorized into three groups according to their weight at birth. The majority of them were in the birth weight group of $2.10-4 \mathrm{~kg}$ amounting to $84.7 \%$ of the sample (Table 1). Investigations regarding the type of marriage of the parents revealed that $73.9 \%$ of the newborns with parents had consanguinity marriage. Regarding the outcome of the surveyed neonates, $70.2 \%$ were born in good health, while $14.1 \%$ were born with morbidity. About the outcome of the affected newborns, $6.1 \%$ of them were referred to specialized centers and $9.6 \%$ were died. Out of 980 neonates with congenital anomalies, only 51 (5.5\%) had head and neck anomalies.

Table 1: Demographic characteristics of the respondents

\begin{tabular}{lcc}
\hline Variables & Frequency & Percentage \\
\hline Anomalies $(n=930)$ & & \\
$\quad$ Head and neck anomalies & 51 & 5.5 \\
$\quad$ Other anomalies & 879 & 94.5 \\
Sex of children $(n=896)$ & & \\
$\quad$ Male & 465 & 51.9 \\
Female & 431 & 48.1 \\
Birth weight $(\mathrm{kg})(n=855)$ & & \\
1-2 & 72 & 8.4 \\
$2.10-4$ & 724 & 84.7 \\
4.10 or more & 5.9 & 6.9 \\
Name of syndrome $(n=926)$ & & \\
Yes & 91 & 9.8 \\
No & 835 & 90.1 \\
Type of marriage $(n=880)$ & & \\
Consanguinity & 650 & 73.9 \\
Non-consanguinity & 230 & 26.1 \\
Outcome $(n=853)$ & & \\
Death & 82 & 9.6 \\
Survive & 599 & 14.1 \\
Referral & 52 & \\
Morbidity & 120 & \\
\hline
\end{tabular}

The incidence rates of head and neck anomalies were calculated among all neonates with anomalies, the highest incidence rate was found in year 1435 (Islamic Hijri Year), in which the incidence rate was $7.1 \%$, followed by 1433 and 1434 (Islamic Hijri Years), 3.17\% and $3.44 \%$ respectively, in comparison with years 1437 and 1432 (Islamic Hijri Years) in which lower incidence rates were found (1.34 and 1.27, respectively), as shown in Table 2 . Cross tabulation between demographic characteristics and head and neck anomalies revealed that sex of the neonates was significantly associated with head and neck anomalies ( $p$ value $=0.015$ ). The birth weight of the surveyed children had no significant association with head and neck anomalies ( $p$ value $=0.148$ ). It could be interpreted as being LBW is not a risk factor of head and neck anomalies. The association between type of marriage and incidence of head and neck anomalies among all anomalies was not significant ( $p$ value $=0.338$ ). Therefore, consanguinity was not found as a risk factor for head and neck anomalies (Table 3).

\section{Discussion}

Congenital head and neck malformations are wide and heterogeneous, as this region comprises part of all organ systems. Such malformations vary merely from cosmetic defects and mild disturbance to deadly abnormalities in their significance and severity. These can be isolated as a part of sequence, syndrome,

Table 2: Incidence rates of head and neck anomalies among the whole live births per year

\begin{tabular}{ll}
\hline Years & Incidence rate (\%) \\
\hline 1432 & 1.27 \\
1433 & 3.71 \\
1434 & 3.44 \\
1435 & 7.1 \\
1436 & 2.07 \\
1437 & 1.34 \\
1438 & 2.06 \\
\hline
\end{tabular}

Table 3: The associations between demographic characteristics of the respondents and incidence of head and neck anomalies among all anomalies

\begin{tabular}{|c|c|c|c|}
\hline \multirow[b]{2}{*}{ Variables } & \multicolumn{2}{|c|}{ Anomalies } & \multirow[b]{2}{*}{ pvalue } \\
\hline & Head and neck (\%) & Others (\%) & \\
\hline \multicolumn{4}{|l|}{ Sex of children } \\
\hline Male & $18(3.9)$ & $447(96.1)$ & 0.015 \\
\hline Female & $32(7.4)$ & $399(92.6)$ & \\
\hline \multicolumn{4}{|l|}{ Birth weight (kg) } \\
\hline $1-2$ & $5(0.06)$ & $67(7.8)$ & 0.148 \\
\hline $2.10-4$ & $4(4.8)$ & $683(79.9)$ & \\
\hline 4.10 or more & $0(0.0)$ & $59(6.9)$ & \\
\hline \multicolumn{4}{|l|}{ Type of marriage } \\
\hline Consanguinity & $615(69.9)$ & $35(4.0)$ & 0.338 \\
\hline Nonconsanguinity & $220(25.0)$ & $10(1.1)$ & \\
\hline \multicolumn{4}{|l|}{ Outcome } \\
\hline Death & $6(0.7)$ & $76(8.9)$ & 0.432 \\
\hline Survive & $30(3.5)$ & $569(66.7)$ & \\
\hline Referral & $4(0.5)$ & $48(5.6)$ & \\
\hline Morbidity & $10(1.2)$ & $110(12.9)$ & \\
\hline
\end{tabular}


or chromosomal disorder. Most of them are genetic in origin, but the rest are caused by teratogens. ${ }^{3}$

Results of this study showed that head and neck anomalies account only $5.5 \%$ among all congenital anomalies (94.5\%). The incidence rate of head and neck anomalies showed a wide variation in different years, with highest rate was found in year 1435 (Islamic Hijri Year), in which the incidence rate was $7.1 \%$. While the lower incidence rate was in year 1432 (Islamic Hijri Year), 1.27\%, the pattern of congenital anomalies and their prevalence may vary over time. Sallout et al. reported that a prevalence of major congenital anomalies was $46.5 / 1,000$ live births (about $4.7 \%)^{3}$ in Saudi Arabia. In India, a study done by Sarkar et al. documented a high prevalence of congenital anomalies (2.22\%) and found the pattern and prevalence of congenital anomalies can differ over time or by location. ${ }^{8}$ Results of this study revealed that head and neck anomalies are common among females, while males have higher percentage among other types of anomalies. These results are similar to a study done in Tanzania by Mashuda et al. in which they mentioned that congenital anomalies are higher among female sex. ${ }^{9}$ However, another study done by Egbe et al. reported no sex difference in the incidence of overall congenital malformations. ${ }^{10}$ There was no significant correlation between congenital anomalies and birth weight in this study. Thus, neonates with LBW are not at a higher risk of congenital anomalies. Moreover, birth weight is an outcome rather than being a predisposing factor. This result was inconsistent with a study done by Mashuda et al. as they found a significant correlation between congenital anomalies and birth weight. ${ }^{9}$

The findings of this study showed no significant correlation between congenital anomalies and consanguinity between parents. This result is inconsistent with the studies done by Al-Hussain and Al-Bunyan and El-Hazmi et al. in which they found a significant association between consanguinity and congenital anomalies. ${ }^{6,7}$ Regarding the outcome of the surveyed children, it is found that $70.2 \%$ of them survive without morbidity, while $14.1 \%$ had a permanent morbidity in their life. Only $9.6 \%$ died while they were in the hospital, and the rest of $6.1 \%$ were referred to continue their treatment in another hospital. Linhart et al. and Cosme et al. reported that congenital anomalies are one of the most significant risk factors for neonatal morbidity and perinatal mortality. 11,12

This study is a retrospective cohort hospital-based study. Data were collected from records, rather than following the surveyed participants. Design of reviewing records might limit the validity of the results. Furthermore, prenatal and genetic factors, which are thought affecting, and may be the predisposing factors to congenital anomalies, were excluded from the study.

\section{Conclusion}

The occurrence of head and neck anomalies showed a wide variation in different years, and the prevalence among other anomalies is associated significantly with sex of newborn.

\section{References}

1. Hunfeld JA, Tempels A, Passchier J, et al. Brief report: parental burden and grief one year after the birth of a child with a congenital anomaly. J Pediatr Psychol 1999;24(6):515-520. DOI: 10.1093/jpepsy/24.6.515.

2. Marina Kos. Head and neck congenital malformations. Acta Clin Croat 2004;43:195-201.

3. Sallout B, Obedat N, Shakeel F, et al. Prevalence of major congenital anomalies at King Fahad Medical City in Saudi Arabia: a tertiary care centre-based study. Ann Saudi Med 2015;35(5):343-351. DOI: 10.5144/0256-4947.2015.343.

4. AlSalloum A, El Mouzan MI, AlHerbish A, et al. Prevalence of selected congenital anomalies in Saudi children: a community-based study. Ann Saudi Med 2015;35(2):107-110. DOI: 10.5144/0256-4947.2015.107.

5. Sàndor GKB, Carmichael RP, Coraza L, et al. Genetic mutations in certain head and neck conditions of interest to the dentist. J Can Dent Assoc 2001;67(10):594-599.

6. Al-Hussain M, Al-Bunyan M. Consanguineous marriages in a Saudi population and the effect of inbreeding on prenatal and postnatal mortality. Ann Trop Paediatric 1997;17(2):155-160. DOI: 10.1080/02724936.1997.11747879.

7. El-Hazmi MA, Al-Swailem AR, Warsy AS, et al. Consanguinity among the Saudi Arabianpopulation. J Med Genet 1995;32(8):623-626. DOI: 10.1136/jmg.32.8.623.

8. Sarkar S, Patra C, Dasgupta MK, et al. Prevalence of congenital Anomalies in neonates and associated risk factors in a tertiary care hospital in eastern India. J Clin Neonatol 2013;2(3):131-134. DOI: 10.4103/2249-4847.119998.

9. Mashuda F, Zuechner A, Chalya PL, et al. Pattern and factors associated with congenital anomalies among young infants admitted at Bugando medical centre, Mwanza, Tanzania. BMC Res Notes 2014;7(1):195-198. DOI: 10.1186/1756-0500-7-195.

10. Egbe A, Uppu S, Lee S, et al. Congenital malformations in the newborn population: a population study and analysis of the effect of sex and prematurity. Pediatr Neonatol 2015;56(1):25-30. DOI: 10.1016/j. pedneo.2014.03.010

11. Linhart Y, Bashiri A, Maymon E, et al. Congenital anomalies are an independent risk factor for neonatal morbidity and perinatal mortality in preterm birth. Eur J Obstet Gynecol Reprod Biol 2000;90(1):43-49. DOI: 10.1016/s0301-2115(99)00196-7.

12. Cosme HW, Lima LS, Barbosa LG. Prevalence of congenital anomalies and their associated factors in newborns in the city of Sao Paulo from 2010 to 2014. Rev Paul Pediatr 2017;35(1):33-38. DOI: 10.1590/19840462/;2017;35;1;00002. 